\title{
Order Parameter Fragmentation after a Symmetry-Breaking Transition
}

\author{
S. Ducci, ${ }^{1, *}$ P. L. Ramazza, ${ }^{1}$ W. González-Viñas, ${ }^{2}$ and F. T. Arecchi ${ }^{1, *}$ \\ ${ }^{1}$ Istituto Nazionale di Ottica, Largo Enrico Fermi, 6, I50125, Florence, Italy \\ and INFM, Unita' di Firenze \\ ${ }^{2}$ Department of Physics and Applied Mathematics, Universidad de Navarra, 31080 Pamplona, Spain
} (Received 26 July 1999)

\begin{abstract}
As a nonlinear optical system consisting of a Kerr medium inserted in a feedback loop is exposed to a light intensity growing linearly from below to above the threshold for pattern formation, the critical slowing down around threshold freezes the defect population. The measured number of defects immediately after the transition scales with the quench time as predicted by Zurek for a two-dimensional Ginzburg-Landau model. The further temporal evolution of the defect number is in agreement with a simple annihilation model, once the drift of defects specific for our system is taken into account.
\end{abstract}

PACS numbers: 05.70.Fh, 05.45.-a, 42.65.Sf, 47.54.+r

Symmetry-breaking transitions are a fundamental subject of research in many fields of physics from condensed matter in thermodynamical equilibrium [1] to nonequilibrium systems that undergo bifurcations [2], possibly leading to patterned states in spatially extended systems [3]. With reference to cosmological models, Kibble [4] stressed that topological defects may have played a fundamental role in the evolution of the early Universe. Zurek [5] captured the general feature of the rapid crossing of the threshold region, pointing out the formal similarity between the cosmological phase transition and some accessible laboratory situations, thus opening the field to a series of theoretical and experimental considerations [6-9]. All these phenomena can be described by a complex field in a quartic potential, as the coefficient of the quadratic term (control parameter) is driven from positive (below threshold) to negative (above threshold) over a quench time $\tau_{q}$. A central issue is the evaluation of the correlation length of the field as a function of $\tau_{q}$ which is the time scale of the control parameter variations. This correlation length has been shown to be given by the average separation of nearest-neighbor topological defects of the field under consideration $[10,11]$. The transient behavior has been modeled and verified in a recent series of papers [12-14] in which the defect number $N$ at the end of the quench time $\tau_{q}$ and its successive evolution have been reported. From an experimental point of view, phase transitions in liquid crystals [15] and in superfluid helium [6-9] have revealed the occurrence of transient phenomena related to defects, but the key prediction of the power-law dependence of the defect number with $\tau_{q}$ has not yet been verified.

In this Letter we report the statistics of defects that form in a two-dimensional (2D) nonlinear optical system as the incident light intensity (control parameter) ramps linearly from below to above the threshold for pattern formation, within a quench time $\tau_{q}$. The initial number $N$ at the end of the quench follows the scaling law $\tau_{q}^{-1 / 2}$ and its successive time evolution is ruled by the combined action of mutual defect annihilation and escape due to drift.
The experimental setup consists of a liquid crystal light valve (LCLV) illuminated by a spatially uniform laser beam and inserted in a feedback loop (see Fig. 1). Within a suitable parameter range, the LCLV acts as an optical Kerr medium. As a result, the beam reflected from the front face of the LCLV undergoes a phase retardation proportional to the light intensity applied to the rear face of the valve. Optical pattern formation in a Kerr-like medium with various types of feedback has been studied both theoretically [16] and experimentally [17]. When the feedback loop includes a free propagation length $L$ introducing diffraction in the optical wave, patterns are due to the interplay of the Kerr effect with the diffusion of the refractive index perturbation and the diffraction. Introduction of a nonlocal interaction by means of a transverse displacement of the optical wave front within the feedback loop induces a new class of pattern-forming instabilities $[18,19]$. The equation for the evolution of the phase of the beam reflected by the LCLV front face is

$$
\begin{aligned}
\tau \frac{\partial u}{\partial t}= & -u(x, y, t)+l_{d}^{2} \nabla_{\perp}^{2} u(x, y, t) \\
& +\alpha I_{\mathrm{fb}}(x+\Delta x, y, t)
\end{aligned}
$$

where $\tau$ and $l_{d}$ are, respectively, the response time and the diffusion length of the liquid crystals, and $(x, y)$ are the coordinates in the plane transverse to the propagation direction $z$. The cell thickness is less than a diffusion length, hence $u$ has no $z$ dependence and $\nabla_{\perp}^{2}$ is the Laplacian operator in the $(x, y)$ plane. $I_{\mathrm{fb}}(x+\Delta x, y, t)$ is the feedback intensity displaced by the amount $\Delta x$ along $x$, and $\alpha$ gives the strength and sign of the Kerr nonlinearity. We use $\alpha$ negative (defocusing case). $I_{\mathrm{fb}}$ is proportional to the input intensity $I_{0}$, which we take as the control parameter, and it includes the effects of dephasing through the cell, diffraction along the propagation length $L$, and lateral displacement $\Delta x$. Calling $I_{0 \text { th }}$ the intensity at the threshold for pattern formation, we rescale $I_{0}$ as $\epsilon \equiv \frac{I_{0}-I_{0 \mathrm{th}}}{I_{0 \mathrm{th}}}$. Close to threshold, the normal form equation for the slowly varying amplitude $A$ of $u$ corresponding to 
Eq. (1) is a complex Ginzburg-Landau equation with the addition of a drift term proportional to the gradient of $A$ along $x$ and to a group velocity $v=2 \alpha I_{0} \Delta x / \tau$.

The experimental results refer to a fixed wavelength $(632 \mathrm{~nm})$ and polarization (parallel to the liquid crystal director) of the incoming beam and the value of the transverse shift is $\Delta x=110 \mu \mathrm{m}$. In these conditions the solution for the intensity pattern is a set of standing rolls perpendicular to $\Delta x$ [18]. While, however, the rolls have zero phase velocity, any local defect is swept away at the group velocity $v$. The symmetry-breaking transition occurs as the input intensity ramps linearly from a value $\epsilon_{i}$ below threshold to a value $\boldsymbol{\epsilon}_{f}$ corresponding to a stable roll-like pattern, within a quench time $\tau_{q}$. The ramp is obtained by passing the input beam through a computercontrolled acousto-optic modulator and the corresponding dynamics is video recorded and successively analyzed. This way we can realize different $\tau_{q}$ 's, thus systematically testing how the defect number $N$ at the bifurcation scales with $\tau_{q}$ and how it decays in the course of time.

We recall the heuristic derivation of the scaling law for $N$ vs $\tau_{q}$ [13]. Consider a 2D field subject to a second order phase transition controlled via a ramp of a parameter $\epsilon$ over a time $\tau_{q}\left(\epsilon=t / \tau_{q}\right.$, where $\epsilon=0$ is the threshold point). The system dynamical response time is $\tau=\tau_{0} / \epsilon, \tau_{0}$ being its inertial time scale. The correlation length is given by $\xi=\xi_{0} / \epsilon^{1 / 2}$, where $\xi_{0}$ is a suitable correlation length far from the transition. When $\epsilon$ is so close to zero that $\tau$ is larger than the time separation $\hat{t}$ from the threshold, the field is no longer able to follow the control parameter variation, due to the critical slowing down. This situation occurs for $t<$ $\sqrt{\tau_{0} \tau_{q}} \equiv \hat{t}$. Hence, in the time interval $-\hat{t}<t<\hat{t}$, the correlation length in the system is frozen at a value $\xi(\hat{t}) \simeq$ $\xi_{0} / \epsilon(\hat{t})^{1 / 2}=\xi_{0}\left(\tau_{q} / \tau_{0}\right)^{1 / 4}$. In a two-dimensional system of size $l$, assuming that a correlation length corresponds to the average separation of nearest-neighbor topological

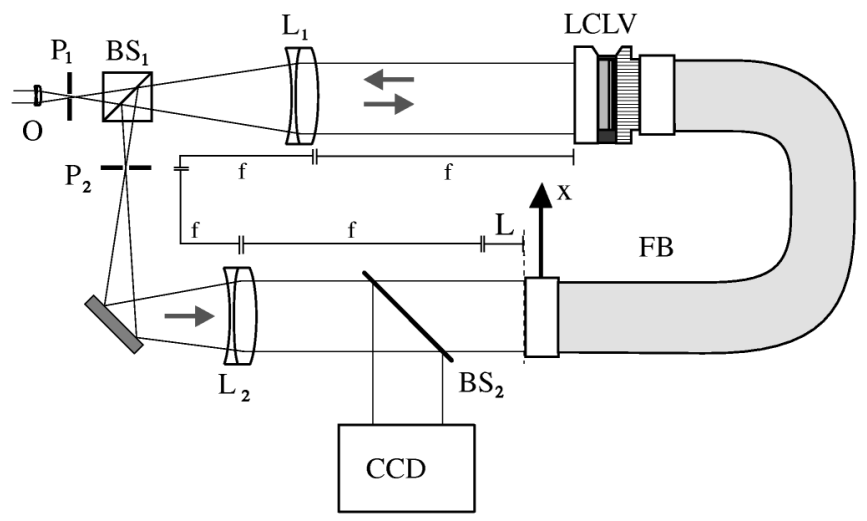

FIG. 1. Experimental setup: $O=$ microscope objective; $P_{1}$, $P_{2}=$ pinholes; $B S_{1}, B S_{2}=$ beam splitters; $L C L V=$ liquid crystal light valve; $L_{1}, L_{2}=$ lenses of focal length $f ; \mathrm{FB}=$ fiber bundle; $X=$ direction of feedback displacement; $C C D=$ videocamera; $L=$ free propagation length (in the experiment $L=3.5 \mathrm{~cm})$. defects, it follows that the number $N$ of defects will scale as $N \simeq(l / \xi)^{2} \propto \tau_{q}^{-1 / 2}$.

A formal similarity between our experimental system and the model of Ref. [13] can be established on the basis that in both cases we are in the presence of a supercritical bifurcation of a complex field. In our case this field represents the slowly varying amplitude of the bifurcating roll pattern; however, due to the nonlocal interaction in the feedback loop, the bifurcated pattern has in general nonzero phase and group velocities. While the former can be set to zero by means of an adequate choice of the operating parameters, the latter is always finite; as a result, the defects in the system are subject not only to annihilation but also to a drift at constant velocity toward one of the boundaries.

Figure 2 is the principal result of our paper; it shows the number of defects measured for various $\tau_{q}$ values. For each value $\tau_{q}$, the pump parameter is varied from $\epsilon_{i}=$ -0.64 to $\epsilon_{f}=0.64$. The threshold $\epsilon=0$ corresponds to $I_{0 \mathrm{th}}=200 \mu \mathrm{W} / \mathrm{cm}^{2}$. The plot is obtained by counting the number of defects produced in each transition at the moment $t_{\text {fin }}=\tau_{q} / 2$ in which the intensity reaches its final value. In our case, as clearly visible in Fig. 3, defects are swept away from the system due to the drift. Precisely, if the whole system is a rectangle of area $h \times l_{0}$ ( $l_{0}$ being parallel to $\Delta x$ and hence to $v$ ), the part of the system in which perturbations to the roll solution (e.g., defects) can survive at the time $t$ is a shrinking rectangle of area $A(t)=h \times l(t), l(t)=\left(l_{0}-v t\right)$. The defect numbers reported in Fig. 2 are corrected in order to be always referred to the whole system area. The straight line is the best fit to $\langle N\rangle=N_{0} \tau_{q}^{-a}$ with $a=0.50 \pm 0.04$. This exponent is in good agreement with the theoretical prediction $a=-1 / 2$. The range of spanned $\tau_{q}$ is limited by the following factors. On the side of short $\tau_{q}$, it makes

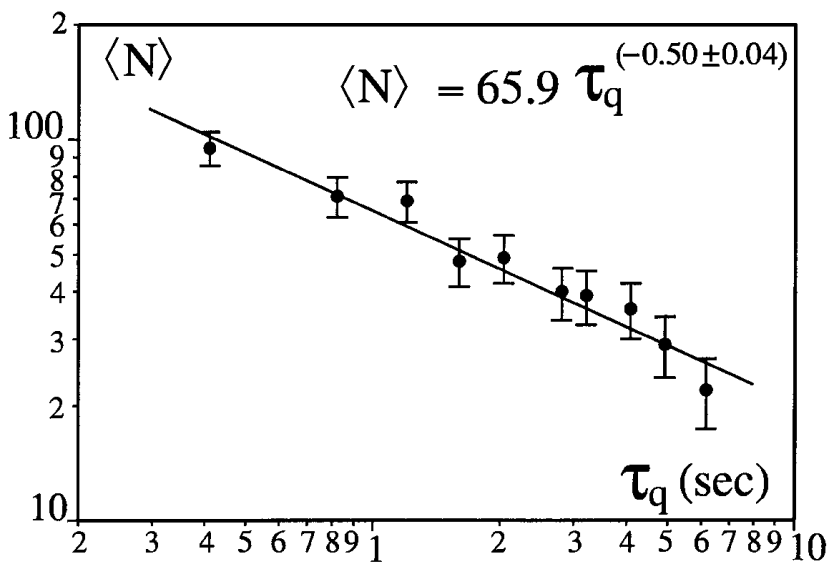

FIG. 2. Defect number obtained in a sequence of phase transitions for various $\tau_{q}$ values. The counting is done at the moment in which the intensity reaches its final value and the results are corrected in order to refer to the whole system area. The straight line is the best fit to $N=N_{0} \tau_{q}^{-a}$ with $a=0.50 \pm 0.04$. 


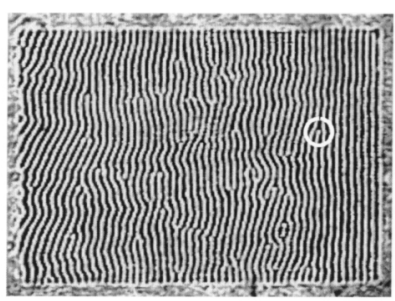

$\mathrm{t}=0.48 \mathrm{~s}$

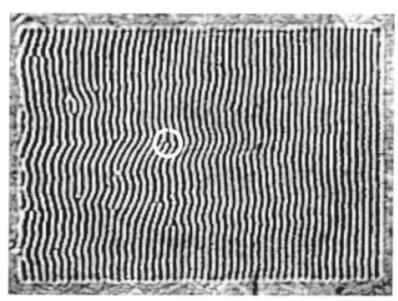

$\mathrm{t}=2.88 \mathrm{~s}$

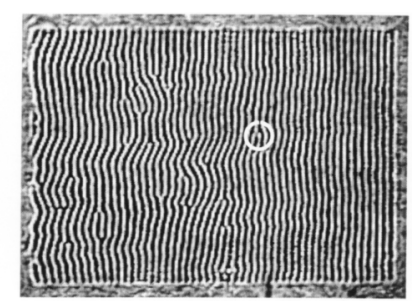

$t=1.92 s$

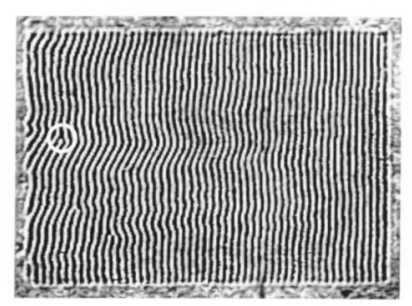

$\mathrm{t}=4.32 \mathrm{~s}$
FIG. 3. Intensity patterns showing the evolution of the number of defects with time for a phase transition with $\tau_{q}=0.41 \mathrm{~s}$. An open circle traces the drift of a defect far enough from other ones, to avoid annihilation within the observation.

no sense to apply a ramp on a time scale faster than, or comparable to, the inertial time scale $\tau_{0}$ that is of the order of $100 \mathrm{~ms}$. On the side of long $\tau_{q}$, it is meaningless to let the system evolve over a time longer than the time $l / v$ over which any perturbation will be swept away by coherent transport. In our experiment the system size is $l_{0}=10.35 \mathrm{~mm}$ and the group velocity $v \simeq 2 \mathrm{~mm} / \mathrm{s}$ for $\epsilon=0.64$, leading to a maximum value of $\tau_{q}$ at about $5 \mathrm{~s}$.

We then measure the evolution of the defect number with time, by counting the defects in a series of images of the type shown in Fig. 3 for the case $\tau_{q}=0.41 \mathrm{~s}$. Since here we are studying the decay of the defect number starting from the end of the quench, that occurs at $t_{\text {fin }}=\tau_{q} / 2$, we take this value as the time origin. In the absence of drift, $N$ decreases because of annihilation processes. The annihilation rate is expected to be of the form $\dot{N}=-\chi N^{2}$, being proportional to the frequency with which defects encounter one another [13]. This leads to the evolution law $N(t)^{-1}=N_{(t=0)}^{-1}+\chi t$, already confirmed in a liquid crystal experiment [15].

In our experiment, the decrease of the defect number in the course of time is due both to the annihilation mechanism with a rate $\dot{N}=-\gamma N^{2} / A(t)$ and to the escape from the boundary at a rate $-N v / l(t)$. We thus have the solution

$$
N(t)=\frac{N_{0}\left(1-v t / l_{0}\right)}{1+\gamma N_{0} t / A(0)},
$$

where $N_{0}$ is the initial number of defects in the area $A(0)$. In Fig. 4 we report the experimental measurements and their relative fits with Eq. (2) for three different values of $\tau_{q}$. The theoretical curves are in good agreement with the experimental data as we can see from the values of $\gamma$ and $v$ reported in the figure caption.

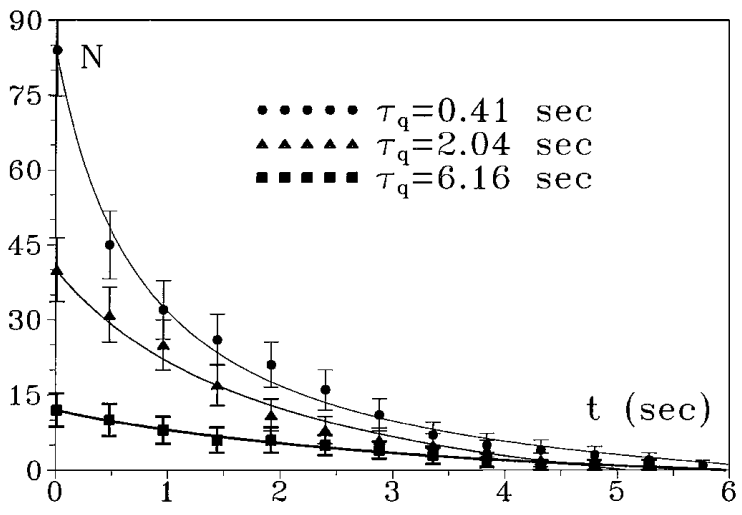

FIG. 4. Experimental measurements (symbols) and relative fits with Eq. (2) (lines) of the temporal evolution of the defect number for three different values of $\tau_{q}$. The best fit values for the parameters $v$ and $\gamma$ result: $v=1.37 \mathrm{~mm} / \mathrm{s}$, $\gamma=0.71 \mathrm{~mm}^{2} / \mathrm{s} ; \quad v=1.63 \mathrm{~mm} / \mathrm{s}, \quad \gamma=0.66 \mathrm{~mm}^{2} / \mathrm{s} ; \quad v=$ $1.1 \mathrm{~mm} / \mathrm{s}, \gamma=0.88 \mathrm{~mm}^{2} / \mathrm{s}$.

In order to have an insight to the range of times over which the process of annihilation dominates, we report also $1 / N$ vs $t$ for $\tau_{q}=0.41 \mathrm{~s}$ in Fig. 5. The plot includes the experimental points, the fit with Eq. (2), and the straight line obtained by putting $v=0$ in Eq. (2). Expanding the expression of $1 / N$ for $t \rightarrow 0$, the quadratic term in $t$ becomes appreciable for $t \geq t^{*}=$ $1.93 \mathrm{~s}$. As we can clearly see in Fig. 4, for $t<t^{*}$ the linear fit is good, meaning that annihilation dominates the drift, thus recovering the results expected in [13] and observed in [15].

In summary, we have studied experimentally the transient statistics of the topological defects in a system with a complex order parameter swept in time across a supercritical bifurcation. Both the dependence of the early defect number on the sweep speed as well as its successive delay rate at later times confirm the theoretical predictions. In the first case, the defect rate dependence upon the sweep

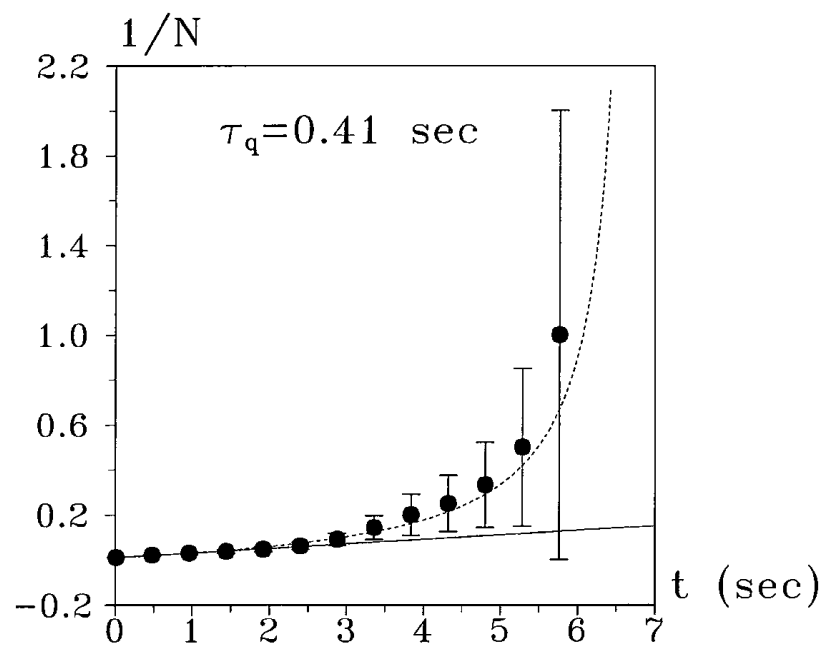

FIG. 5. $1 / N$ vs $t$ for $\tau_{q}=0.41 \mathrm{~s}$ : experimental data (dots), fit with Eq. (2) (dashed line), and straight line obtained by putting $v=0$ in Eq. (2). 
time is a power law with a $-1 / 2$ exponent. In the second case, once we account for a drift peculiar to our system, the defect annihilation model, based on a mass-action-like law, results are confirmed.

This work has been partly supported by ECC Contract No. FMRX CT960010 and the 1999 Italy-Spain Integrated Action.

*Also at Dept. of Physics, University of Florence, Florence, Italy.

[1] For a comprehensive review of early results in this field, see, e.g., P. C. Hohenberg and B. I. Halperin, Rev. Mod. Phys. 49, 435 (1977).

[2] See, e.g., Dynamical Critical Phenomena and Related Topics, edited by C.P. Enz (Springer-Verlag, Berlin, 1979).

[3] M. C. Cross and P.C. Hohenberg, special issue, Pattern Formation Outside of Equilibrium, Rev. Mod. Phys. 65 (1993).

[4] T. W. B. Kibble, J. Phys. A 9, 1387 (1976); Phys. Rep. 67, 183 (1980).

[5] W. H. Zurek, Nature (London) 317, 505 (1985).

[6] P. C. Hendry, N.S. Lawson, R. A. M. Lee, P. V.E. McClintock, and C.H.D. Williams, Nature (London) 368, 315 (1994); V.M.H. Ruutu, V.B. Eltson, A.J. Gill, T.W.B. Kibble, M. Krusius, Y.G. Makhlin, B. Placais, G. E. Volovik, and Wen Xu, Nature (London) 382, 334 (1996).

[7] M. E. Dodd, P. C. Hendry, N. S. Lawson, P. V. E. McClintock, and C.D.H. Williams, Phys. Rev. Lett. 81, 3703
(1998); G. Karra and R. J. Rivers, Phys. Rev. Lett. 81, 3707 (1998); D. I. Bradley, S. N. Fisher, and W. M. Hayes, J. Low Temp. Phys. 113, 687 (1998).

[8] G. Karra and R. J. Rivers, Phys. Rev. Lett. 81, 3707 (1998).

[9] D. I. Bradley, S. N. Fisher, and W. M. Hayes, J. Low Temp. Phys. 113, 687 (1998).

[10] P. Coullet, L. Gil, and J. Lega, Phys. Rev. Lett. 62, 1619 (1989).

[11] F. T. Arecchi, G. Giacomelli, P. L. Ramazza, and S. Residori, Phys. Rev. Lett. 67, 3749 (1991).

[12] P. Laguna and W.H. Zurek, Phys. Rev. Lett. 78, 2519 (1997).

[13] A. Yates and W.H. Zurek, Phys. Rev. Lett. 80, 5477 (1998).

[14] N.D. Antunes, L. M. A. Bettencourt, and W.H. Zurek, Phys. Rev. Lett. 82, 2824 (1999).

[15] I. Cuang, R. Durrer, N. Turok, and B. Yurke, Science 251, 1336 (1991).

[16] S.A. Akhmanov, M. A. Vorontsov, and V. Yu. Ivanov, JETP Lett. 47, 707 (1998); W. Firth, J. Mod. Opt. 37, 151 (1990).

[17] R. MacDonald and H. J. Eichler, Opt. Commun. 89, 289 (1992); S. Akhmanov, M. A. Vorontsov, V. Yu. Ivanov, A. V. Larichev, and N. I. Zheleznykh, J. Opt. Soc. Am. B 9, 78 (1992); P. L. Ramazza, E. Pampaloni, S. Residori, and F. T. Arecchi, Physica (Amsterdam) 96D, 259 (1996), and references therein.

[18] P. L. Ramazza, S. Boccaletti, A. Giaquinta, E. Pampaloni, S. Soria, and F. T. Arecchi, Phys. Rev. A 54, 3472 (1996).

[19] P. L. Ramazza, S. Ducci, and F. T. Arecchi, Phys. Rev. Lett. 81, 4128 (1998). 